\title{
Injeção intravítrea de cetorolaco de trometamina em pacientes com edema macular diabético refratário à fotocoagulacão retiniana
}

\author{
Intravitreal injection of ketorolac tromethamine in patients with diabetic macular edema \\ refractory to retinal photocoagulation
}

Andreia do Ceu Afonso Reis ${ }^{1}$, Raul Nunes Galvarro Vianna ${ }^{2}$, Ricardo Siqueira Mendes dos Reis ${ }^{1}$, Gilberto Perez Cardoso $^{3}$

\section{RESUMO}

Objetivo: Avaliar o efeito em curto prazo da injeção intravítrea de cetorolaco de trometamina $(500 \mathrm{\mu g} / 0,1 \mathrm{ml})$ em pacientes com edema macular diabético refratário à fotocoagulação retiniana.

Métodos: Estudo prospectivo. Foram selecionados 20 pacientes com edema macular diabético refratário à fotocoagulação retiniana em ambos os olhos e apresentando acuidade visual (ETDRS) entre 20/50 e 20/200. Foi injetado em um olho de cada paciente, pela via intravítrea, $500 \mu \mathrm{g} \mathrm{em} \mathrm{0,1} \mathrm{ml} \mathrm{de} \mathrm{cetorolaco}$ de trometamina, sem conservante. O olho contralateral serviu de controle. Exames oftalmológicos, que incluíram a acuidade visual (ETDRS) com melhor correção, a aferição da pressão intraocular e a tomografia de coerência óptica, foram realizados em 3 etapas: antes da aplicação, após uma semana e posteriormente após um mês do tratamento.

Resultados: Houve diferença estatisticamente significativa na acuidade visual (ETDRS) ao longo do tempo, havendo uma melhora na visão do olho tratado $(p=0,039)$ quando comparado com o olho contralateral. Não foram encontradas diferenças estatisticamente significativas na avaliação da pressão intraocular $(p=0,99)$, espessura foveal $(p=0,86)$ e volume macular total $(p=0,23)$ no decorrer do período

Conclusão: Pacientes com edema macular diabético refratário à fotocoagulação apresentaram melhora da acuidade visual ao longo de um mês com diferença estatisticamente significativa quando comparados com o olho controle. Não foram encontradas diferenças estatisticamente significativas na pressão intraocular, espessura foveolar e volume macular total entre os olhos tratados e os olhos controle.

Descritores: Retinopatia diabética/quimioterapia; Injeções; Corpo vítreo Pressão intraocular; Edema macular/quimioterapia; Retina/efeito de drogas; Cetorolaco de trometamina/administração \& dosagem; Coagulação por laser; Acuidade visual

\section{ABSTRACT}

Purpose: To evaluate the effect of a single dose of intravitreous injection of ketorolac tromethamine $(500 \mathrm{\mu g} / 0.1 \mathrm{ml})$ in patients with diabetic macular edema refractory to retinal photocoagulation.

Methods: Prospective study. Twenty patients with bilateral diabetic macular edema and ETDRS best-corrected visual acuity between 20/50 and 20/200 were selected. Patients who had other ocular diseases or previous eye surgery were excluded. Preservative-free ketorolac tromethamine was injected intravitreally (500 $\mathrm{mg}$ in $0.1 \mathrm{ml}$ ) in 20 eyes; fellow eyes served as controls. Ophthalmic examinations included ETDRS best-corrected visual acuity measurement of intraocular pressure and optical coherence tomography. The examinations were performed preoperatively, 1 week and 1 month postoperatively.

Results: A statistically significant increase in visual acuity over time in the treated eye compared with the fellow eye was noted $(p=0.039$ ). There were no statistically significant differences in the assessment of intraocular pressure $(p=0.99)$, foveal thickness $(p=0.86)$ and macular volume $(p=0.23)$ during the period.

Conclusion: Patients with diabetic macular edema refractory to photocoagulation showed improvement in visual acuity over a one month period with a statistically significant difference when compared with the control eye. There were no statistically significant differences in intraocular pressure, foveolar thickness and macular volume between the treated and control eyes.

Keywords: Diabetic retinopathy/drug therapy; Injections; Vitreous body; Intraocular pressure; Macular edema/drug therapy; Retina/drug effects; Ketorolac tromethanine/ administration \& dosage; Laser coagulation; Visual acuity
Trabalho realizado na Disciplina de Oftalmologia, Universidade Federal Fluminense - UFF Niterói (RJ), Brasil.

${ }^{1}$ Médico, Ciências Médicas, Universidade Federal Fluminense - UFF - Rio de Janeiro (RJ), Brasil.

Médico, Serviço de Oftalmologia, Universidade Federal Fluminense - UFF - Rio de Janeiro (RJ), Brasil.

${ }^{3}$ Médico, Clínica Médica, Universidade Federal Fluminense - UFF - Rio de Janeiro (RJ), Brasil. Endereço para correspondência: Andreia do Ceu Afonso Reis. Rua Francisco Sá, 23 - Sala 1.207 - Rio de Janeiro (RJ) - CEP 22080-010

E-mail:afonsoandreia@ig.com.b

Recebido para publicação em 07.10.2009

Última versão recebida em 17.05.2010

Aprovação em 12.07.2010

0 presente trabalho teve o auxílio da Fundação de Amparo à Pesquisa do Estado do Rio de Janeiro - FAPERJ.

\section{INTRODUÇÃO}

0 edema macular (EM) é a principal causa de baixa visual em pacientes diabéticos. A fotocoagulação a laser é o tratamento convencional nesses casos e reduz o risco de perda visual moderada (definido como perda de 15 ou mais letras na tabela ETDRS) em aproximadamente $50 \%$ dos olhos tratados. No entanto, os outros $50 \%$ apresentam declínio da visão ao longo do tempo, apesar do tratamento ${ }^{(1-2)}$. Assim, é fundamental que se pesquisem novas terapias que venham a melhorar os resultados morfológicos e funcionais para esses pacientes.

A utilização de fotocoagulação com micropulso tem sido uma alternativa pois a terapia com laser menos destrutiva com uma razão risco-benefício mais favorável pode justificar o tratamento mais precoce, possibilitando a melhora ou estabilização de uma função visual menos comprometida ${ }^{(3)}$.

Atualmente, já está estabelecido o papel da inflamação na patogênese do edema macular diabético $(E M D)^{(4)}$. Dessa forma, existe uma lógica para a utilização de corticosteroides, 
tanto pelas vias perioculares como pelas intraoculares, para a sua terapia. De fato, o acetato de triancinolona (AT), a dexametasona e o acetato de fluocinolona, quando utilizados pelas vias previamente citadas, tem demonstrado bons resultados na redução do EMD. Contudo, os efeitos adversos (catarata e glaucoma), que são comuns aos três, limitam em muito os seus usos ${ }^{(5)}$.

Recentemente, medicamentos inibidores do fator de crescimento do endotelio vascular (Vascular Endothelial Growth Factor-VEGF) têm emergido como uma nova abordagem no tratamento de complicações da retinopatia diabética (RD). Entretanto, embora administrado pela via intravítrea, o medicamento anti-VEGF pode passar para a circulação sistêmica, o que poderia resultar em hipertensão, proteinúria, aumento da ocorrência de eventos cardiovasculares e dificuldade de cicatrização. Por este motivo, estudos para avaliar não só a eficácia, mas também a sistemática dos efeitos adversos dessas drogas em uma população de alto risco, como diabéticos, são necessários, e ainda estão sendo realizados ${ }^{(6)}$. Além disso, as drogas inibidoras do VEGF liberadas pelo órgão regulador de medicamentos no Brasil (Agência Nacional de Vigilância Sanitária ANVISA) tem um alto custo, o que também dificulta a sua utilização para uma população de baixa renda.

Considerando as evidências que mostram que a inibição da ciclo-oxigenase e derivados da prostaglandinas podem reduzir o metabolismo e as anormalidades histológicas da retinopatia diabética ${ }^{(7)}$, os anti-inflamatórios não-esteroides estão sendo estudados para atuar no tratamento do EMD. Um estudo experimental conduzido por alguns autores ${ }^{(8)}$, em 2006, reportaram que não houve retinotoxicidade na administração intraocular do cetorolaco de trometamina (CT) em coelhos, na dosagem de $500 \mu \mathrm{g}$. Em humanos, outros autores ${ }^{(7)}$, também em 2006, demonstraram que houve redução da espessura retiniana e melhora da acuidade visual em olhos com EMD e EM por outras retinopatias, como oclusão de veia central da retina, após a administração intravítrea de CT em dosagens que variaram entre 60 e $900 \mu \mathrm{g}$. Ademais, além do baixo custo, uma grande vantagem dos anti-inflamatórios não-esteroides é que os mesmos não são cataratogênicos e não causam aumento da pressão intraocular ${ }^{(8)}$.

Diante desta possibilidade, decidimos estudar prospectivamente os efeitos em curto prazo da injeção intravítrea de CT na acuidade visual e na morfologia macular de pacientes com EMD refratário à fotocoagulação com laser de argônio.

\section{MÉTODOS}

O projeto desta pesquisa foi aprovado pelo Comitê de Ética Médica e Pesquisa da Faculdade de Medicina/Hospital Universitário Antonio Pedro, Universidade Federal Fluminense.

\section{Pacientes}

Os 20 pacientes deste estudo foram selecionados no Serviço de Oftalmologia do Hospital Universitário Antonio Pedro, da
Universidade Federal Fluminense. Todos os pacientes apresentavam EMD no exame de biomicroscopia de retina em ambos os olhos e acuidade visual (AV) corrigida entre 20/50 e 20/200 na tabela ETDRS. Após o consentimento livre e esclarecido, os pacientes foram submetidos a exame oftalmológico completo, que inclui: anamnese, medida da AV corrigida pela tabela ETDRS (tabela de distância de 4,5 metros), tonometria de aplanação, biomicroscopia de retina e vítreo com lente +66 D (Volk Super 66, JH0759, EUA) e exame de tomografia de coerência óptica (OCT) (Zeiss OCT - 3000 - Stratus 4.0, EUA). O olho com pior AV foi eleito para o tratamento.

\section{Protocolo}

O exame de OCT foi realizado com o aparelho Zeiss OCT 3000 -Stratus 4.0, utilizando o modo Fast de Macular Thickeness Map, sob midríase medicamentosa. O OCT é um exame de alta resolução, não-invasivo e de não-contato, que usa luz com comprimento de onda próximo ao infravermelho, para produzir imagens seccionais da retina, em sentido sagital. Fornece imagem quantitativa e qualitativa da retina, assemelhando-se a um corte histológico e que permite avaliar a espessura retiniana, a espessura foveal (EF), o volume macular total (VMT) e a camada de fibras nervosas ${ }^{(9)}$. Para o exame de AV foi utilizada a tabela ETRDS logMAR a distância de 4,5 metros e com correção da refração. A pressão intraocular (PIO) foi aferida com tonômetro de aplanação de Goldmann (Zeiss).

O CT 0,5\% é registrado, para uso tópico, no Ministério da Saúde e comercializado com o nome de Acular ${ }^{\circledR}$ sem conservante -flaconetes, pela Allergan. O olho selecionado para tratamento era aquele com melhor AV. A injeção intravítrea do CT foi realizada no centro cirúrgico e foi administrada a dosagem de $500 \mu \mathrm{g}$ de $C T$, em 0,1 ml na agulha de insulina.

A AV, a tonometria de aplanação e a OCT foram realizadas antes da aplicação, bem como após 7 e 30 dias posteriores a aplicação intravítrea da medicação.

\section{AnÁlise estatística}

A análise estatística foi realizada pelos seguintes métodos:

- para analisar o comportamento das variáveis ao longo do tempo (três momentos) separadamente por grupo de olho foi realizada a Análise de Variância para medidas repetidas. O teste de comparações múltiplas de Bonferroni (ajustado para medidas repetidas) foi aplicado para identificar quais os momentos que diferem entre si; e

- para verificar se o comportamento ao longo do tempo é diferenciado entre os grupos de olhos foi realizada a Análise de Variância para medidas repetidas com um fator.

O critério de determinação de significância adotado foi o nível de 5\%. A análise estatística foi processada pelo software SAS 6.04 (SAS Institute, Inc., Cary, NC).

Tabela 1. Análise longitudinal da AV por olho

\begin{tabular}{lcccccccc}
\hline Olho & Momento & $\mathbf{n}$ & Média & DP & Mediana & Mínimo & Máximo & $\boldsymbol{p ~ v a l o r}^{\boldsymbol{a}}$ \\
\hline Tratado & Pré-tto & 20 & 0,161 & 0,080 & 0,12 & 0,1 & 0,32 & 0,039 \\
& 1 sem. após & 20 & 0,186 & 0,104 & 0,12 & 0,1 & 0,40 & \\
& 1 mês após & 20 & 0,193 & 0,118 & 0,12 & 0,1 & 0,40 & \\
Controle & Pré-tto & 20 & 0,209 & 0,137 & 0,11 & 0,1 & 0,50 & 0,37 \\
& 1 sem. após & 20 & 0,205 & 0,139 & 0,11 & 0,1 & 0,50 & \\
& 1 mês após & 20 & 0,204 & 0,139 & 0,10 & 0,1 & 0,50 & \\
\hline
\end{tabular}

$\mathrm{DP}=$ desvio padrão; $p$ valor ${ }^{\mathrm{a}}=\mathrm{ANOVA}$ para medidas repetidas para o logaritmo da AV; Pré-tto= pré-tratamento 
Tabela 2. Análise longitudinal da PIO (mmHg) por olho

\begin{tabular}{lcccccccc}
\hline Olho & Momento & $\mathbf{n}$ & Média & DP & Mediana & Mínimo & Máximo & $\boldsymbol{p ~ v a l o r ~}^{\boldsymbol{a}}$ \\
\hline Tratado & Pré-tto & 20 & 16,0 & 2,4 & 16 & 12 & 20 & 0,99 \\
& 1 sem. após & 20 & 16,0 & 2,6 & 16 & 12 & 22 & \\
\multirow{2}{*}{ Controle } & 1 mês após & 20 & 15,9 & 2,2 & 15 & 12 & 20 & \\
& Pré-tto & 20 & 16,0 & 2,2 & 16 & 12 & 20 & 0,90 \\
& 1 sem. após & 20 & 15,9 & 2,3 & 16 & 12 & 20 & \\
& 1 mês após & 20 & 15,9 & 2,1 & 15 & 12 & 20 & \\
\hline
\end{tabular}

$\mathrm{DP}=$ desvio padrão; $p$ valor ${ }^{\mathrm{a}}=\mathrm{ANOVA}$ para medidas repetidas para o logaritmo da PIO; Pré-tto= pré-tratamento

Tabela 3. Análise longitudinal da EF (m) por olho

\begin{tabular}{lcccccccc}
\hline Olho & Momento & $\mathbf{n}$ & Média & DP & Mediana & Mínimo & Máximo & $\boldsymbol{p ~ v a l o r}^{\boldsymbol{a}}$ \\
\hline Tratado & Pré-tto & 20 & 367,9 & 188,7 & 345,5 & 173 & 810 & 0,86 \\
& 1 sem. após & 20 & 362,4 & 191,0 & 316 & 142 & 771 & \\
& 1 mês após & 20 & 369,4 & 180,2 & 334 & 152 & 680 & \\
Controle & Pré-tto & 20 & 448,8 & 272,7 & 454 & 143 & 1082 & 0,36 \\
& 1 sem. após & 20 & 412,8 & 241,0 & 346 & 146 & 980 & \\
& 1 mês após & 20 & 414,8 & 230,7 & 421 & 147 & 898 & \\
\hline
\end{tabular}

$\mathrm{DP}=$ desvio padrão; $p$ valor ${ }^{2}=$ ANOVA para medidas repetidas para o logaritmo da EF; Pré-tto= pré-tratamento

Tabela 4. Análise longitudinal da VMT $\left(\mathrm{mm}^{3}\right)$ por olho

\begin{tabular}{lcccccccc}
\hline Olho & Momento & $\mathbf{n}$ & Média & DP & Mediana & Mínimo & Máximo & $\boldsymbol{p ~ v a l o r}^{\boldsymbol{a}}$ \\
\hline Tratado & Pré-tto & 20 & 9,65 & 2,62 & 8,59 & 6,80 & 15,98 & 0,23 \\
& 1 sem. após & 20 & 9,48 & 2,51 & 8,45 & 6,67 & 15,04 & \\
& 1 mês após & 20 & 9,43 & 2,43 & 8,45 & 6,67 & 15,31 & \\
\multirow{2}{*}{ Controle } & Pré-tto & 20 & 10,67 & 4,48 & 9,39 & 6,47 & 23,87 & 0,82 \\
& 1 sem. após & 20 & 10,59 & 4,12 & 9,43 & 6,46 & 20,03 & \\
& 1 mês após & 20 & 10,85 & 4,84 & 9,72 & 6,47 & 25,27 & \\
\hline
\end{tabular}

$\mathrm{DP}=$ desvio padrão; $p$ valor $r^{\mathrm{a}}=$ ANOVA para medidas repetidas para o logaritmo do VMT; Pré-tto= pré-tratamento

\section{RESULTADOS}

As tabelas 1, 2, 3 e 4 fornecem a média, desvio padrão (DP), mínimo, máximo da AV, PIO, EF e VMT, respectivamente, para três momentos (pré-tratamento, 1 semana após e 1 mês após) de cada olho, e o correspondente nível descritivo (valor de p) da Análise de Variância para medidas repetidas.

Pela Análise de Variância para medidas repetidas, observouse que existe variação significativa na AV ao longo do tempo apenas no olho tratado $(p=0,039)$ (Tabela 1$)$.

Pela Análise de Variância para medidas repetidas, observou-se que não existe variação significativa na PIO ao longo do tempo no olho tratado $(p=0,99)$ e nem no olho controle $(p=0,90)$ (Tabela 2). Também não existe variação significativa na EF ao longo do tempo no olho tratado $(p=0,86)$ e nem no olho controle $(p=0,36)$ (Tabela 3$)$. E em relação ao VMT não existe variação significativa ao longo do tempo no olho tratado $(p=0,23)$ e nem no olho controle $(p=0,82)$ (Tabela 4$)$.

A Análise de Variância para medidas repetidas com um fator verifica a influência do olho, do tempo e da interação (olho*tempo) nas variáveis. O quadro 1 fornece o resultado da ANOVA para as variáveis (AV, PIO, EF e VMT). Os valores na tabela correspondem ao nível de significância ( $p$ valor) de cada componente (olho, tempo e interação) da ANOVA.

A Análise de Variância para medidas repetidas com um fator mostrou que na AV:

- não existe variação significativa ao longo do tempo $(p=0,21)$
- existe diferença na evolução (delta entre dois momentos) da AV entre os olhos $(p=0,014)$.

Concluiu-se que, a evolução da AV deu-se de forma diferenciada entre os dois olhos. Não existiu diferença significativa na curva da PIO, EF e VMT entre os dois olhos estudados.

\section{DISCUSSÃO}

A patogênese do EMD é complexa e multifatorial e os detalhes específicos da patogênese do edema macular diabético ainda não são bem esclarecidos ${ }^{(10)}$.

O AT têm sido usados, no intuito de bloquear as vias da inflamação em casos de EMD ${ }^{(11)}$. Porém, a eficácia da terapia com AT e a segurança na utilização em casos onde se faz necessário repetir aplicações para o tratamento do EMD ainda está sendo estabelecida ${ }^{(5)}$.

Os medicamentos inibidores do VEGF têm sido uma opção para o tratamento, mas, como já mencionado, faltam pesquisas em pacientes diabéticos não só em relação à eficácia, como também, relacionadas aos efeitos adversos.

Anti-inflamatórios não-esteroides podem oferecer uma viável, potencial e segura alternativa. Em 2008 alguns autores estudaram a eletrorretinografia e a histopatologia do CT intravítreo, na dosagem de 3000 mg, e revelaram que estes são bem toleráveis e seguros na retina de coelhos e não foi detectado no vítreo após 48 horas da aplicação, porém foram encontrados efeitos contínuos na retina após ter sido absorvido por aproximadamente 8 semanas ${ }^{(12)}$. 
Analisando-se a tabela 1, observa-se que houve diferença estatisticamente significativa na AV entre os dois olhos ao longo do tempo, demonstrando que o CT a 0,5\% contribuiu para um melhora da AV obtida em uma semana e mantida ao longo de um mês.

A tabela 2 demonstra que não houve diferença estatisticamente significativa na análise da PIO longitudinal entre os dois olhos. Em 2006 um trabalho relatou que o CT injetado intravítreo não causou aumento da PIO em coelhos e o mesmo fol constatado nesta pesquisa, mostrando que ao contrário dos anti-inflamatórios esteroides, os anti-inflamatórios não-esteroides não causam aumento da PIO o decorrer do tempo ${ }^{(8)}$

As tabelas 3 e 4 também não demonstraram diferença estatisticamente significativa apesar da AV ter melhorado, o VMT e o EF praticamente se mantiveram altos (Figura 1A,1B, 1C) o que nos faz levantar a hipótese de que apesar de não ter sido observado uma diminuição anatômica do EMD houve uma melhora funcional, com a melhora da AV podendo ter sido causado por um diminuição do estímulo inflamatório e/ou um rearranjo da camada de fotorreceptores.

O Quadro 1 reforça o resultado de que a AV apresentou-se de forma diferenciada entre o olho tratado e o olho controle, havendo melhora significativa da AV entre o pré-tratamento e após um mês de tratamento apenas no olho tratado. Não existiu diferença significativa na curva da PIO, EF e VMT entre os dois olhos estudados.

\section{CONCLUSÃO}

É importante ressaltar que este é um estudo piloto e por se tratar de uma doença de causas multifatoriais acreditamos que o tratamento com anti-inflamatórios não-esteroides possam se agregar ao nosso arsenal terapêutico em associação ou de forma isolada. Estudos com um número maior de pacientes, diferentes doses e posologias do $\mathrm{CT}$, bem como maior tempo de seguimento são desejáveis para melhor avaliação da eficácia e segurança dessa modalidade terapêutica.
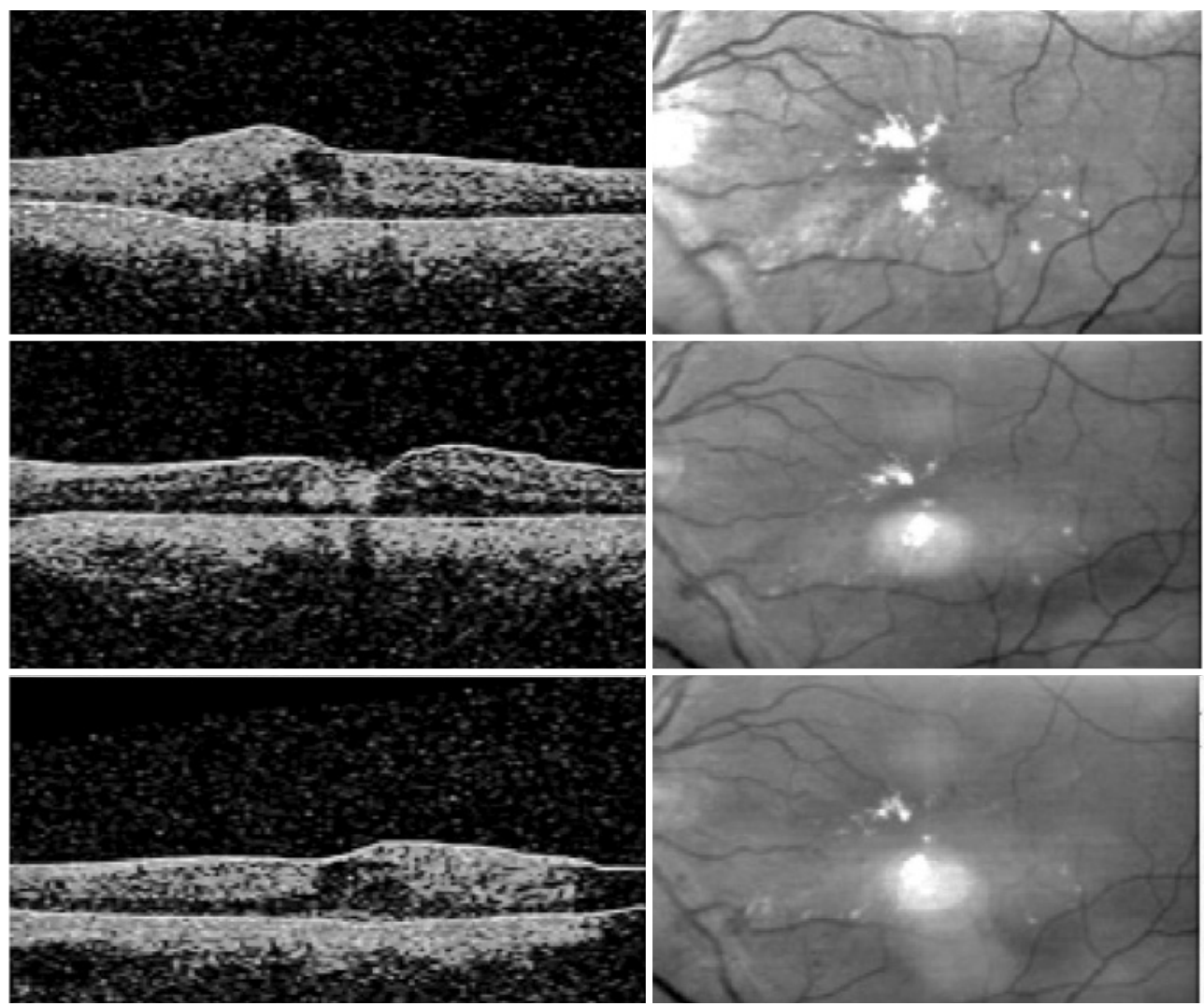

Figura 1.Tomografia de coerência óptica mostrando o EMD antes da injeção intravítrea de CT (A), com 1 semana (B) e 1 mês após a injeção (C).

Quadro 1. ANOVA para medidas repetidas com um fator (logaritmo das variáveis)

\begin{tabular}{|c|c|c|c|}
\hline \multirow[b]{2}{*}{$\begin{array}{l}\text { Variável } \\
\text { (logaritmo) }\end{array}$} & \multicolumn{3}{|c|}{ Componentes } \\
\hline & \begin{tabular}{l}
\multicolumn{2}{c}{ Olho } \\
(tratado $\times$ controle)
\end{tabular} & $\begin{array}{c}\text { Tempo } \\
\text { (pré, } 1 \text { sem., } 1 \text { mês após) }\end{array}$ & $\begin{array}{c}\text { Interação } \\
\text { (olho*tempo) }\end{array}$ \\
\hline AV & 0,70 & 0,21 & 0,014 \\
\hline $\mathrm{PIO}$ & 0,97 & 0,92 & 0,96 \\
\hline$E F$ & 0,54 & 0,43 & 0,71 \\
\hline VMT & 0,42 & 0,66 & 0,46 \\
\hline
\end{tabular}




\section{REFERÊNCIAS}

1. Early Treatment Diabetic Retinopathy Study Research Group. Treatment techniques and clinical guidelines for photocoagulation of diabetic macular edema. Ophthalmology. 1987:94(7):761-74

2. Weinreb RN, Kashiwagi K, Kashiwagi F. Prostaglandins increased matrix metalloproteinase release from human ciliary smooth muscle cells. Invest Ophthalmol Vis Sci. 1997:38(13):2772-80

3. Dare A, Castro L, Lavinsky D, Navajas E, Cardillo JÁ. Novos horizontes no tratamento do edema de macula diabético: fotocoagulaçâo macular seletiva com micropulso de diodo 810nm. Retina e Vítreo. 2007:13:16-20.

4. Joussen AM, Smyth N, Niessen C. Pathophysiology of diabetic macular edema. Dev Ophthalmol. 2007:39:1-12.

5. Furlani BA, Meyer CH, Rodrigues EB, Maia M, Farah ME, Holz FG. Emerging pharmacotherapies for diabetic macular edema. Expert Opin Emerg Drugs. 2007 12(4):591-603.

6. Simó R, Hernández C. Intravitreous anti-VEGF for diabetic retinopathy: hopes and fears for a new therapeutics strategy. Diabetologia. 2008;51(9):1574-80.
7. Wafapoor H, McCluskey J. Intravitreal ketorolac as a treatment of diabetic and nondiabetic macular edema. Invest Ophthalmol Vis Sci. 2006;47:E-abstract 4227.

8. Margalit E, Kugle LJ, Brumm MV, Meza JL, Kompella UB, Escobar ER, et al. The safety of intraocular ketorolac in rabbits. Invest Ophthalmol Vis Sci. 2006;47(5): 2093-9.

9. Sánchez-Tocino H, Alvarez-Vidal A, Maldonado MJ, Moreno-Montañés J, GarcíaLayana A. Retinal thickness study with optical coherence tomography in patients with diabetes. Invest Ophthalmol Vis Sci. 2002;43(5):1588-94.

10. Bhagat N, Grigorian RA, Tutela A, Zarbin MA. Diabetic macular edema: pathogenesis and treatment. Surv Ophthalmol. 2009;54(1):1-32.

11. Yilmaz T, Weaver CD, Gallagher MJ, Cordero-Coma M, Cervantes-Castaneda RA Klisovic D, et al. Intravitreal triamcinolone acetonide injection for treatment of refractory diabetic macular edema: a systematic review. Ophthalmology. 2009; 116(5): 912-3.

12. Kim ST, Adams NA, Toma HS, Belair ML, Thorne JE, Green WR, et al. Safety of intravitreal ketorolac and diclofenac: an electroretinographic and histopathologic study. Retina. 2008;28(4):595-605.

\title{
13ํㅡㅁ Congresso de Oftalmologia USP
}

\author{
$12^{\circ}$ Congresso de Auxilliar \\ de Oftalmologia USP
}
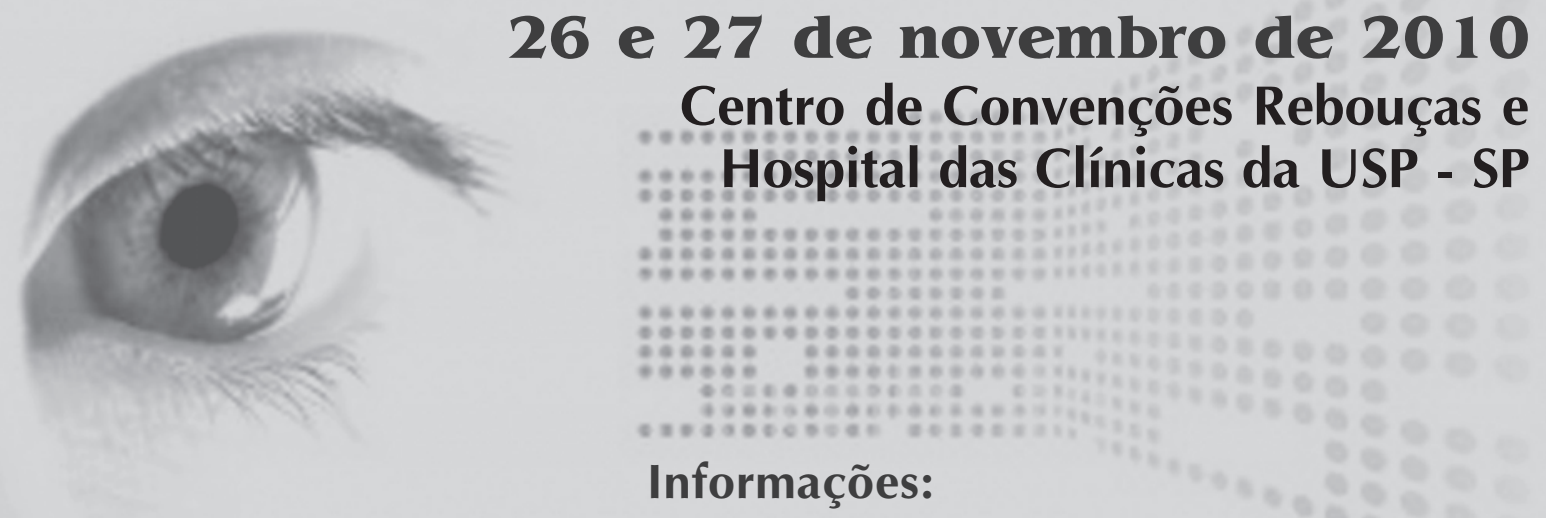

JDE Comunicação e Eventos

Tels: (11) 5082-3030 / 5084-9174

Site: www.oftalmologiausp.com.br 